\title{
Novel gradient echo sequence-based amide proton transfer magnetic resonance imaging in hyperacute cerebral infarction
}

\author{
DEXIAO HUANG, SHENKAI LI, ZHUOZHI DAI, ZHIWEI SHEN, GEN YAN and RENHUA WU \\ Department of Medical Imaging, The Second Affiliated Hospital, Medical College of Shantou University, \\ Shantou, Guangdong 515041, P.R. China
}

Received March 13, 2014; Accepted November 25, 2014

DOI: $10.3892 / \mathrm{mmr} .2015 .3165$

\begin{abstract}
In the progression of ischemia, $\mathrm{pH}$ is important and is essential in elucidating the association between metabolic disruption, lactate formation, acidosis and tissue damage. Chemical exchange-dependent saturation transfer (CEST) imaging can be used to detect tissue $\mathrm{pH}$ and, in particular, a specific form of CEST magnetic resonance imaging (MRI), termed amide proton transfer (APT) MRI, which is sensitive to $\mathrm{pH}$ and can detect ischemic lesions, even prior to diffusion abnormalities. The critical parameter governing the ability of CEST to detect $\mathrm{pH}$ is the sequence. In the present study, a novel strategy was used, based on the gradient echo sequence (GRE), which involved the insertion of a magnetization transfer pulse in each repetition time (TR) and minimizing the TR for in vivo APT imaging. The proposed GRE-APT MRI method was initially verified using a tissue-like $\mathrm{pH}$ phantom and optimized MRI parameters for APT imaging. In order to assess the range of acute cerebral infarction, rats $(n=4)$ were subjected to middle cerebral artery occlusion (MCAO) and MRI scanning at 7 telsa (T). Hyperacute ischemic tissue damage was characterized using multiparametric imaging techniques, including diffusion, APT and $\mathrm{T}_{2}$-Weighted MRI. By using a magnetization transfer pulse and minimizing TR, GRE-APT provided high spatial resolution and a homogeneous signal, with clearly distinguished cerebral anatomy. The GRE-APT and diffusion MRI were significantly correlated with lactate content and the area of cerebral infarction in the APT and apparent diffusion coefficient (ADC) maps matched consistently during the hyperacute period. In addition, compared with the infarction area observed on the ADC MRI map, the APT map contained
\end{abstract}

Correspondence to: Professor Renhua Wu, Department of Medical Imaging, The Second Affiliated Hospital, Medical College of Shantou University, 22 Xin Lin Road, Shantou, Guangdong 515041, P.R. China

E-mail:rhwu@stu.edu.cn

Key words: hyperacute ischemia, amide proton transfer, chemical exchange saturation transfer, $\mathrm{pH}$, stroke tissue, which had not yet been irreversibly damaged. Therefore, GRE-APT MRI waa able to detect ischemic lactic acidosis with sensitivity and spatiotemporal resolution, suggesting the potential use of $\mathrm{pH}$ MRI as a surrogate imaging marker of impaired tissue metabolism for the diagnosis and prognosis of hyperacute stroke.

\section{Introduction}

Ischemic stroke is a severe disease, which often results in mortality or disability. Previous studies on ischemic stroke have demonstrated that thrombolytic treatment increased the survival rate and reduced disability in stroke patients (1). However, early intervention, which stimulates the reperfusion of ischemic penumbra is essential for effective therapeutic outcomes following ischemic stroke $(1,2)$. Therefore, the availability of non-invasive imaging technologies, which are able to rapidly and specifically evaluate ischemic penumbra, are required for the progression of therapeutic research into acute stroke. The penumbra has been previously defined as a large area of metabolically-impaired perfusion deficit, which is able to retain cellular polarization $(3,4)$. In the last 20 years, perfusion-weighted imaging (PWI) and diffusion-weighted imaging (DWI) in magnetic resonance imaging (MRI) have become the most well-established imaging techniques for detecting regions of reduced blood flow and cytotoxic edema, respectively $(5,6)$. Furthermore, PWI/DWI mismatch is considered to represent ischemic tissue, which has not yet undergone severe tissue damage and has been used surgically to define the ischemic penumbra (7-9). However, ischemic tissue damage is complex and multifactorial and PWI/DWI mismatch provides only an approximation of the penumbra $(10,11)$. In addition, the diagnostic accuracy of PWI/DWI mismatch remains controversial. Therefore, novel imaging techniques are required to augment existing penumbral imaging, provide greater insight into disease pathophysiology and assist in guiding treatment decisions.

Ischemic stroke induces a complex cascade of metabolic disruption in the cerebral tissue, which alters oxygen and glucose metabolism, tissue acidosis, adenosine triphosphate depletion and, ultimately, cerebral infarction (12-15). During ischemia, anaerobic metabolism and the formation of lactate 
lead to a decreased $\mathrm{pH}$ in the ischemic area. The disruption of metabolic processes may then be further exacerbated by hypoperfusion and the reduced buffering capacity of bicarbonate at an acidic $\mathrm{pH}$, which may result in further tissue acidification $(16,17)$. As energy metabolism is essential for cell viability and is disrupted at levels of blood flow that are higher than those that cause infarction, monitoring tissue metabolism may offer additional insights into early ischemia prior to irreversible damage (18).

As the chemical exchange between amide protons and bulk tissue water is $\mathrm{pH}$-dependent, amide proton chemical exchange saturation transfer (CEST) MRI is able to assess tissue $\mathrm{pH}$ in vivo (19-23). In particular, a specific form of CEST MRI, termed amide proton transfer (APT) MRI, has been identified as sensitive to $\mathrm{pH}$ and can detect ischemic lesions prior to diffusion abnormalities (2,23-27). However, the sensitivity of CEST MRI requires enhanced due to the weak CEST effects (28-35). The sequence and optimization of parameters are the key features of the CEST experiments to focus on in the improvement of CEST sensitivity. The present study used a novel strategy based on the gradient echo sequence (GRE) combined with magnetic transfer and an in vivo rat acute cerebral infarction model was used to detect the ischemic penumbra using GRE-APT MRI.

\section{Materials and methods}

Phantom preparation. A multi-pH CEST gel phantom composed of creatine and low gelling point (LGP) agarose (Sigma-Aldrich, St. Louis, MO, USA) was prepared, as previously described (20). In brief, $1 \%$ agarose was added to phosphate-buffered saline (PBS; Oxoid, Basingstoke, UK). The phantom was microwave-heated to boiling and then cooled for $\sim 25$ mins in a water bath set at $50^{\circ} \mathrm{C}$ (Cole-Palmer, Vernon Hills, IL, USA). Creatine (Sigma-Aldrich) was then added to the gel solution to final concentrations of 50, 100 and $200 \mathrm{mM}$, followed by serial titration to $\mathrm{pH} 7.0$ (EuTech Instruments, Ayer Raja, Singapore). The solutions were transferred into separate $10 \mathrm{ml}$ centrifuge tubes for each creatinine concentration, which were then sealed and inserted into a phantom holder (63 mm Swift Cradle; Agilent Technologies, Inc., Santa Clara, CA, USA). A central tube filled with $1 \%$ agarose-gel in the absence of creatine was used as a background control.

Acute stroke animal model. The study was approved by the ethics committee of the Second Affiliated Hospital of Shantou University Medical College, Guangdong, China. The protocol was approved by the Institutional Animal Care and Use Committee. Adult male Sprague-Dawley (SD) rats $(n=4)$ aged 7-8 weeks (190-210 g) were obtained from Shantou University Medical College Laboratory Animal Center (Shantou, China) and kept in a $10 / 14 \mathrm{~h}$ light/dark cycle at $22-26^{\circ} \mathrm{C}$ with access to $30-40 \mathrm{~g}$ rat diet and $60-70 \mathrm{ml}$ water per day. The rats underwent imaging within $0.5-3 \mathrm{~h}$, and then again $24 \mathrm{~h}$ following permanent filament middle cerebral artery occlusion (MCAO), while remaining under $\sim 1.5 \%$ isoflurane-induced anesthesia (Shandong Keyuan Pharmaceutical Co., Ltd, Jinan, China), as previously described (2). The heart rate and blood oxygen partial pressure of the rats were monitored online using a real-time Nonin Pulse Oximeter 8600 (Nonin Medical, Inc.,
Plymouth, MN, USA). Standard permanent MCAO was induced by inserting a 4-0 nylon suture (Monosof 4-0; Tyco Healthcare, Zaltbommel, Netherlands) into the lumen of the internal carotid artery in order to block the origin of the middle cerebral artery. Following MRI using an Agilent 7.0 T animal MRI scanner (Agilent Technologies, Inc.) during the period of acute ischemia between 0.5 and $3 \mathrm{~h}$, the animals were revived and provided with free access to food and water. The same anesthesia procedure was then repeated for a $24 \mathrm{~h}$ post MCAO follow-up MRI. $\mathrm{T}_{2}$-weighted hyperintensity, which has previously been confirmed as a reliable approach (33), was used to quantify the levels of infarction by comparing the abnormal MR signal from the MCAO side to that of the normal brain tissue.

MRI parameters. Using an Agilent 7.0 T animal MRI scanner (Agilent Technologies, Inc.), the in vitro scanning parameters, including repetition time (TR) value, echo time (TE) and flip angle (FA) values of the gradient echo (GRE); FA value and duration time $(\mathrm{T})$ of the presaturation pulse and the average readout direction $\mathrm{x}$ phase-encoding direction (ROxPE), were assessed to determine the optimal GRE parameter for hyperacute cerebral infarction. All experiments were performed using an Agilent 7T/160 animal scanner with a volume radiofrequency $(\mathrm{RF})$ coil. The routine imaging parameters were set as follows: FA, 20 ${ }^{\circ}$ TR, minimum $26.6 \mathrm{~ms}$; TE, minimum $2.31 \mathrm{~ms}$; slice thickness, $2 \mathrm{~mm}$; field of view, $60 \times 60 \mathrm{~mm}$; number of averages, 1 and acquisition bandwidth, $50 \mathrm{kHz}$. A GRE sequence with magnetization transfer was used. The presaturation time was $20 \mathrm{~ms}$ and the FA was $\sim 180^{\circ}$. The total scan duration changed according to the matrix (frequency coding number $x$ phase coding number) which determines the resolution of the MR image. A duration of only $2.6 \mathrm{sec}$ was required for imaging with a $64 \times 64 \mathrm{~mm}$ matrix. All images were processed using the intrinsic VnmrJ 3.1A workstation (Agilent Technologies, Inc.) and Matlab, version 7.13 (Mathworks, Natick, MA, USA).

Methodfor CEST imaging. CEST imaging was processed using asymmetric analysis. Three images were acquired, as follows: A normal T1 image without RF offset $\left(\mathrm{I}_{0}\right)$; a T1 image with $\mathrm{RF}$ offset at the resonance of the labile protons $\left(\mathrm{I}_{\mathrm{lib}} ; 3.5 \mathrm{ppm}\right)$ and a T1 image with RF offset at the negative resonance point relative to water $\left(\mathrm{I}_{\text {ref }} ;-3.5 \mathrm{ppm}\right)$. The CEST imaging $\left(\mathrm{I}_{\mathrm{CEST}}\right)$ was then calculated using the following formula (20):

$$
\mathrm{I}_{\mathrm{CEST}}=\left(\mathrm{I}_{\mathrm{ref}}-\mathrm{I}_{\mathrm{lib}}\right) / \mathrm{I}_{0}
$$

\section{Results}

Determination of the Z-spectrum. The APT MRI method was initially verified using a tissue-like $\mathrm{pH}$ phantom to optimize the APT imaging parameters at 7.0 T MRI (36). The Z-spectrum, obtained through monitoring the tissue-like $\mathrm{pH}$ phantom, presented a clear CEST effect at $\sim 1.87 \mathrm{ppm}(516 \mathrm{~Hz}$ at $7.0 \mathrm{~T}$ ) (Fig. 1A). In addition, the CEST effect and signal intensity of the phantom tubes was positively correlated with creatine concentration (Fig. 1B). The GRE-APT MRI measurements were markedly altered in global ischemia. As shown in Fig. 2, the Z-spectrum of the SD rats was obtained in vivo by detecting the tissue water signal at 7.0 T MRI, 
A

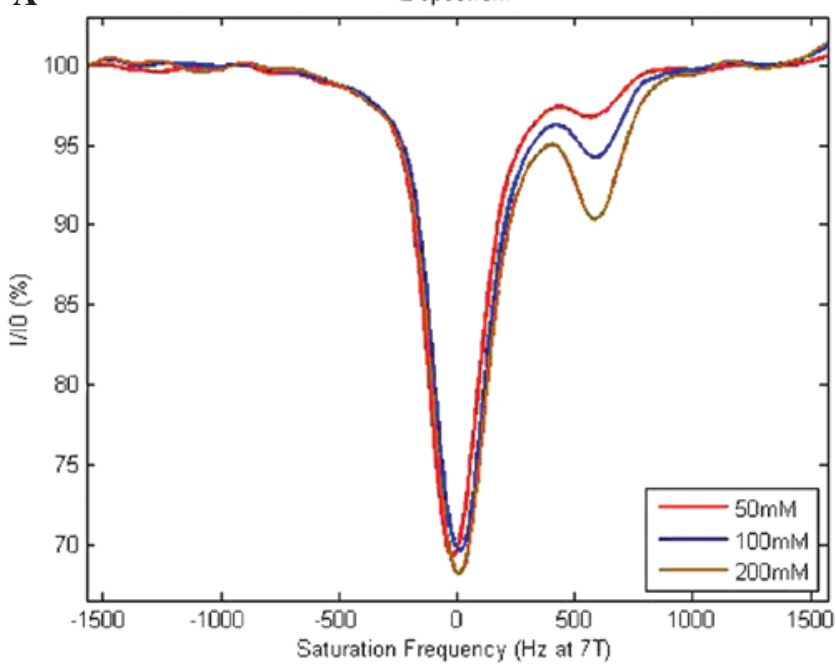

B

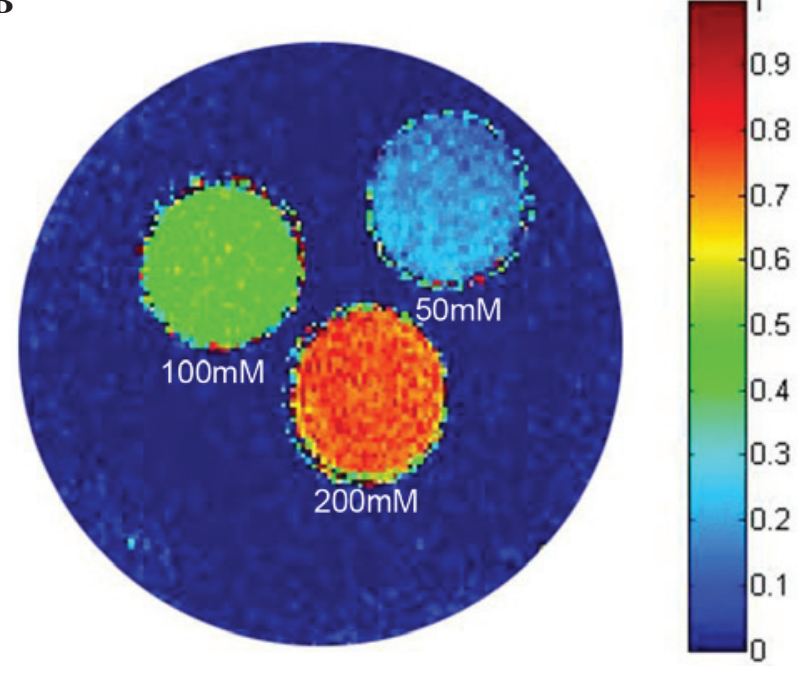

Figure 1. Z-spectrum obtained by monitoring a tissue-like $\mathrm{pH}$ phantom (A) A marked CEST effect was identified at $1.87 \mathrm{ppm}(516 \mathrm{~Hz}$ at $7.0 \mathrm{~T})$. (B) CEST effect and signal intensity of phantom tubes were positively correlated with creatine concentration. CEST, chemical exchange-dependent saturation transfer.

following optimization of the MRI parameters, including FA, TR, TE, slice thickness, field of view, number of excitations and acquisition bandwidth, for sensitizing the proton exchange of the endogenous proteins/peptides. The maximal change in Z-spectral intensity was observed at $3.5 \mathrm{ppm}(1,050 \mathrm{~Hz}$ at $7.0 \mathrm{~T}$ ), which was obtained through processing with a fat saturated frequency (Fsatfrq) function and corresponded to specific amide protons representing a composite amide proton chemical shift. This indicated that tissue $\mathrm{pH}$ can be assessed and imaged using GRE-APT MRI. A change in the Z-spectra was also observed a change at $-3.5 \mathrm{ppm}$, where the CEST effect was $94.4 \%$ on the normal side of the cerebrum and increased to $95.6 \%$ on the ischemic side during the hyperacute period. This was consistent with the hypothesis that endogenous amide proton exchange occurred primarily due to changes in intracellular $\mathrm{pH}$ (Fig. 2).

APT MRI of normal SD rats. In a pilot APT MRI investigation of normal SD rats $(\mathrm{n}=3)$ in vivo, the APT imaging was

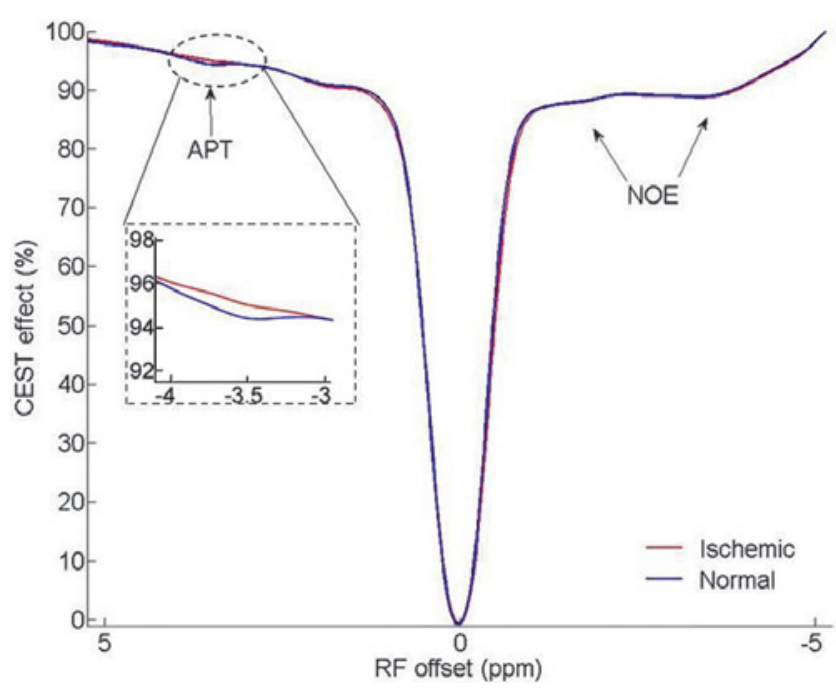

Figure 2. A Z-spectrum was obtained by monitoring the tissue water signals of Sprague-Dawley rats using 7.0 T magnetic resonance imaging in vivo. The maximal change in Z-spectral intensity was observed at $\sim 3.5 \mathrm{ppm}(1,050 \mathrm{~Hz}$ at $7.0 \mathrm{~T}$ ), which represented a composite amide proton chemical shift. The NOE indicates the transfer of nuclear spin polarization from one nuclear spin population to another, via cross-relaxation. CEST, chemical exchange-dependent saturation transfer; RF, radiofrequency; APT, amide proton transfer; NOE, nuclear Overhauser enhancement effect.

calculated as the magnetization transfer ratio (MTR) asymmetry: $\mathrm{I}_{\mathrm{ref}} \mathrm{I}_{\mathrm{libl}} / \mathrm{I}_{0}$, in which $\mathrm{I}_{\text {ref }}$ and $\mathrm{I}_{\text {lib }}$ denote the reference and labile protons, respectively, with RF applied at -3.5 and $3.5 \mathrm{ppm}(1,050 \mathrm{~Hz}$ at $7.0 \mathrm{~T}) . \mathrm{I}_{0}$ denotes the scan without $\mathrm{RF}$ irradiation (Fig. 3). Fig. 3A is an MT image obtained with RF applied at -3.5 and $3.5 \mathrm{ppm}(1,050 \mathrm{~Hz}$ at $7.0 \mathrm{~T})$, and without RF irradiation. Fig. 3B shows the APT imaging obtained with RF irradiation and via processing with Vnmr J 3.1A and Matlab software. This method of imaging provided high spatial resolution and a homogeneous signal, which provided an appropriate resolution to distinguish the cerebral anatomy. The GRE-APT MRI appeared reasonably homogeneous within the cerebrum and may be applied for the detection of subtle $\mathrm{pH}$ lesions in hyperacute stroke.

APT MRI of SD rats following the induction of MCAO. All four MCAO rats observed in the present study demonstrated large deficits across the relatively hypoperfused region serviced by the right MCA, with corresponding deficits on the APT and apparent diffusion coefficient (ADC) maps (Fig. 4). However, no abnormal signals were observed on the $T_{1}$ or $T_{2}$-weighted images during the hyperacute period between 0.5 and $3.0 \mathrm{~h}$. Furthermore, the deficits in the APT and ADC maps matched consistently during the hyperacute period, indicating severe energy breakdown and concomitant cell depolarization. All the hypoperfused regions progressed to complete infarction, which was confirmed by a 24 h-post-MCAO follow-up $\mathrm{T}_{2} \mathrm{WI}$. Furthermore, the deficits on the APT and ADC maps were marginally increased during the hyperacute period. At 24 h-post-MCAO, the infarcted areas in the T2-weighted images corresponded with those of the hyperacute deficits on the APT maps. However, GRE-APT MRI may provide further insight into infarcted areas compared with ADC MRI. Unlike the ADC map, there were certain imaging characteristics with 


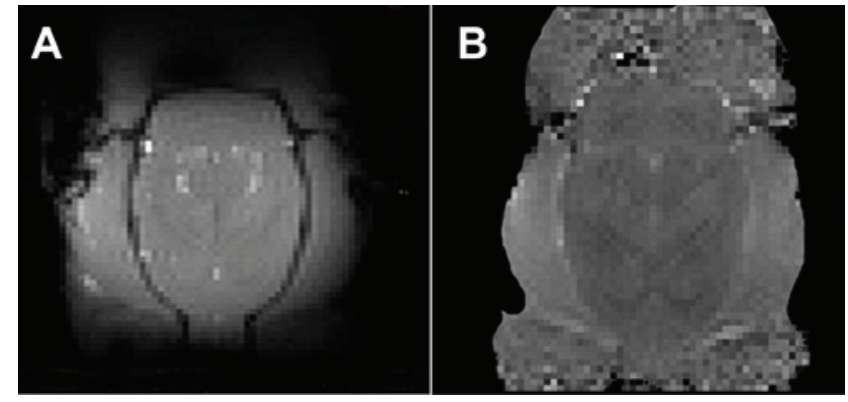

Figure 3. pH-weighted APT magnetic resonance imaging of a normal subject at 7.0 T. (A) MT image obtained with RF applied at -3.5 and $3.5 \mathrm{ppm}(1,050 \mathrm{~Hz}$ at 7.0 T), and without RF irradiation. (B) The APT imaging was obtained with RF irradiation and via processing with Vnmr J 3.1A and Matlab software, which provide high spatial resolution and a homogeneous signal, and enable resolution of the cerebrum anatomy. APT, amide proton transfer; RF, radio frequency.
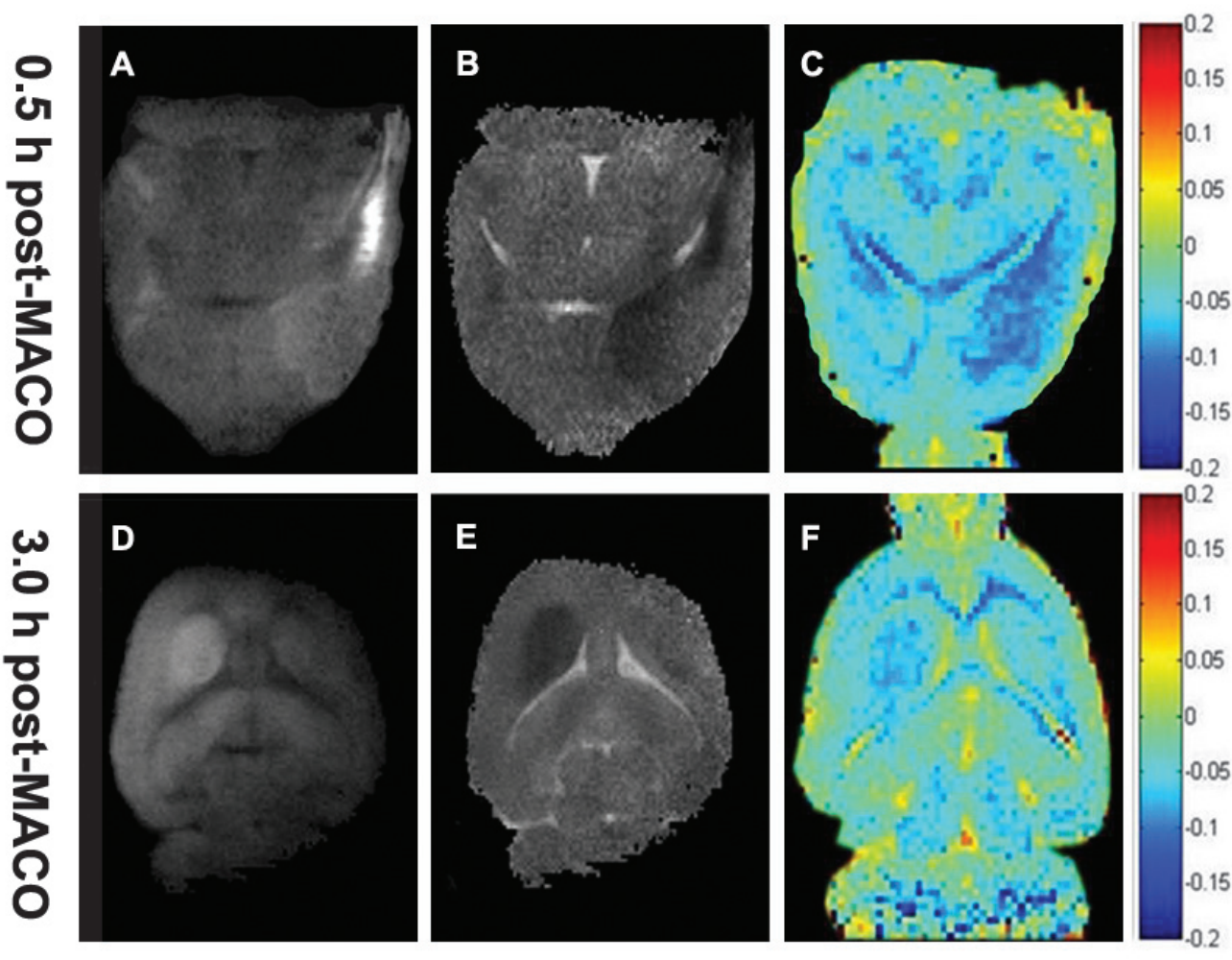

DWI

ADC

APT

Figure 4. DWI and $\mathrm{pH}$-weighted endogenous APT MRI of a representative hyperacute stroke (between $0.5 \mathrm{~h}$ and $3.0 \mathrm{~h}$ post-MCAO) animal. DWI, ADC and APT MRI images of rats (A-C) $0.5 \mathrm{~h}$ and (D-F) $3 \mathrm{~h}$ post-MCAO, respectively. Deficits of the APT and ADC maps matched consistently during the hyperacute period. Unlike the ADC map, the APT map exhibited an undefined deficit boundary and a gradient difference in lesion signal, with marked signal in the center and weak signal in the periphery during the hyperacute period. APT, amide proton transfer; MRI, magnetic resonance imaging; MCAO, middle cerebral artery occulsion; ADC, apparent diffusion coefficient; DWI, diffusion-weighted imaging.

undefined deficit boundaries on the APT map and the gradient of hypointensity ranged, being marked in the center and weak in the peripheries during the hyperacute period. This observation may indicate that the infarction area on the ADC map contained tissue that had not undergone irreversible damage, as demonstrated in the imaging characteristics of the APT map.

\section{Discussion}

CEST imaging, a novel molecular imaging technology, is able to non-invasively characterize the physical and physiological status of tissues in vivo, including tissue $\mathrm{pH}, \mathrm{APT}$, glycogen, glycosaminoglycan, lipid, enzymes and genes $(28,37)$. The present study demonstrated that the sensitivity of CEST MRI was significantly improved by inserting a magnetization transfer pulse at each TR and by minimizing TR, based on the GRE sequence. As the effect of the pulses was cumulative, the CEST effect was enhanced and reached a steady state following multiple pulses. In addition, the present study verified this strategy using the tissue-like CEST phantoms at 7.0 T. In the Z-spectrum, the CEST effect was proportional to the concentration of the labile protons and the CEST effect increased with the phase-encoding number within a certain 
range, which suggested that this proposed novel method may enhance the effectiveness of pH-weighted APT MRI. Therefore, pH-weighted APT/CEST MRI based on a GRE sequence combined with magnetic transfer was capable of detecting ischemic lactic acidosis and provided APT imaging with sensitivity and spatiotemporal resolution, which was consistent with previous studies $(21,26,38)$. In the present study, deficits were observed in the APT and ADC maps, which matched consistently and increased marginally during the hyperacute period. At 24 h-post-MCAO, the infarct area in the T2-weighted images corresponded with that of the APT map deficits observed between 0.5 and $3.0 \mathrm{~h}$. In addition, compared with that of the conventional CEST imaging sequences (39-42), the method proposed in the present study demonstrated certain advantages, including a markedly reduced scan time, improving the temporal resolution of the APT imaging and enabling the imaging of patients with only minimal or no interference with their clinical care. In addition, the proposed method extended the interval of the saturation pulses, leading to a highly reduced specific absorption rate and enabling use of the interval to obtain a signal. Multislice imaging is also easily obtained. Overall, the method proposed by the present study may be easily translated in order to obtain CEST imaging on the majority of clinical MRI machines. The goal of future studies is to fully develop $\mathrm{pH}$-weighted APT MRI and to evaluate its diagnostic use to ultimately augment the current diagnostic capability of hyperacute stroke.

The optimization of multiple MRI parameters is essential to improve the evaluation of ischemic tissue metabolism for predicting tissue outcomes and their response to potential treatments. Notably, pH-sensitive MRI may be beneficial in patients with tissues that are not irreversibly damaged. Previous animal studies have demonstrated that there may be a significant $\mathrm{CBF} / \mathrm{pH} / \mathrm{ADC}$ mismatch in acute ischemic stroke and that $\mathrm{pH}$-weighted APT lesions may be used to improve the prediction of tissue outcomes (2). In addition, $\mathrm{pH}$-weighted MRI may be able to delineate the PWI/DWI mismatch into a benign oligemic region and metabolic penumbra. However, the conventional CEST imaging sequence used for APT MRI in vivo provides only $\mathrm{pH}$-weighted information. GRE-APT MRI using the modified sequence has been shown to provide additional insight into areas of infarction compared with ADC MRI and conventional CEST imaging sequences (39-42). The present study revealed APT map imaging characteristics with undefined deficit boundaries and a hypointensity gradient, which was more marked at the center and weaker at the peripheries during the hyperacute period. This indicated that the infarct area observed on the ADC map contained tissue which had not yet undergone irreversible damage. Within this $\mathrm{pH}$-impaired region, it is likely that the metabolism was completely interrupted at the center and initially sustained at the peripheries. This may have been due to anaerobic glycolysis during the hyperacute period, leading to the accumulation of lactic acid and a reduction in local tissue $\mathrm{pH}$, as indicated by the gradient of hypointensity in the APT MRI. The relatively weak signals in the lesion peripheries compared with those at the center may represent an area of impaired metabolism, which remained viable on the APT map, despite its identification as an infarct on the ADC map. These findings may be extended to the use of $\mathrm{pH}$ MRI as a surrogate imaging marker of impaired tissue metabolism and suggested that $\mathrm{pH}$-weighted APT MRI may be used for the clinical diagnosis and determination of prognosis in patients with acute stroke.

The regulation of $\mathrm{pH}$ is important in the progression of disease, therefore, it is essential to elucidate the association between metabolic disruption, lactate content, acidosis and tissue damage. Various techniques have been developed for $\mathrm{pH}$-weighted APT imaging in vivo, however, improvements are to provide higher sensitivity, signal-to-noise ratio and concentration independence (43). A pH-weighted APT MRI offers a more adaptable approach as exchange sites may be built into the agent molecules to make them concentration independent (44). In the present study, APT MRI was used to identify and characterize lesions in a permanent stroke model to examine the association between tissue $\mathrm{pH}$, diffusion and lactic acidosis in acute shock. However, APT MRI may be extended to other diseases, including solid tumors and disorders of glycogen, glycosaminoglycan, lipid, enzymes and genes.

In conclusion, the present study characterized heterogeneous ischemic tissue damage using multiparametric MRI of $\mathrm{pH}$, diffusion and relaxation imaging during the hyperacute period. A novel method was used, based on the GRE sequence combined with magnetic transfer and phase encoding, which demonstrated that $\mathrm{pH}$-weighted APT provided high spatial resolution, a homogeneous signal and clearly distinguished cerebral anatomy. In addition, the $\mathrm{pH}$-weighted APT and diffusion MRI were significantly correlated with lactate content and the deficits of the APT and ADC maps matched consistently during the hyperacute period. The GRE-APT MRI provided further insight into the infarction area compared with the ADC MRI, as reflected in certain lesions on the APT map exhibiting undefined deficit boundaries and a hypointensity gradient, which was more marked at the center and weaker at the peripheries during the hyperacute period. This indicated the presence of tissue within the penumbra, which had not yet undergone irreversible damage. Furthermore, the present study demonstrated that, due to its high sensitivity to tissue acidification, pH-weighted APT MRI using the gradient echo sequence provided surrogate imaging markers of impaired tissue metabolism. This suggested that $\mathrm{pH}$-weighted APT MRI may be used for clinical diagnosis and for determining prognosis in patients with hyperacute stroke.

\section{References}

1. Ciccone A, Valvassori L, Nichelatti M, et al: Endovascular treatment for acute ischemic stroke. N Engl J Med 368: 904-913, 2013.

2. Sun PZ, Zhou J, Sun W, Huang J and van Zijl PCM: Detection of the ischemic penumbra using pH-weighted MRI. J Cereb Blood Flow Metab 27: 1129-1136, 2007.

3. Astrup J: Thresholds of cerebral ischemia. In: Microsurgery for stroke (Schmiedek P, ed), Berlin: Springer, 16-21, 1976.

4. Hossmann KA: Viability thresholds and the penumbra of focal ischemia. Ann Neurol 36: 557-565, 1994.

5. Hjort N, Butcher K, Davis SM, et al: Magnetic resonance imaging criteria for thrombolysis in acute cerebral infarct. Stroke 36: 388-397, 2005.

6. Wu O, Christensen S, Hjort N, et al: Characterizing physiological heterogeneity of infarction risk in acute human ischaemic stroke using MRI. Brain 129: 2384-2393, 2006.

7. Warach S: Thrombolysis in stroke beyond three hours: targeting patients with diffusion and perfusion MRI. Ann Neurol 51: $11-13,2002$. 
8. Schlaug G, Benfield A, Baird AE, et al: The ischemic penumbra: Operationally defined by diffusion and perfusion MRI. Neurology 53: 1528-1537, 1999.

9. Schaefer PW, Ozsunar Y, He J, et al: Assessing tissue viability with MR diffusion and perfusion imaging. Am J Neuroradiol 24: 436-443, 2003.

10. Kidwell CS, Alger JR and Saver JL: Beyond mismatch: evolving paradigms in imaging the ischemic penumbra with multimodal magnetic resonance imaging. Stroke 34: 2729-2735, 2003.

11. Sobesky J, Weber OZ, Lehnhardt F-G, Hesselmann V, Neveling M, Jacobs A and Heiss W-D: Does the mismatch match the penumbra? Magnetic resonance imaging and positron emission tomography in early schemic stroke. Stroke 36: 980-985, 2005.

12. Astrup J, Siesjö BK, Symon L, et al: Thresholds in cerebral ischemia-the ischemic penumbra. Stroke 12: 723-725, 1981.

13. Siesjö BK: Pathophysiology and treatment of focal cerebral ischemia: Part I: Pathophysiology. J Neurosurg 77: 169-184, 1992.

14. Hossmann KA: Viability thresholds and the penumbra of focal ischemia. Ann Neurol 36: 557-565, 1994

15. An H, Liu Q, Chen Y, Lin W, et al: Evaluation of MR-Derived cerebral oxygen metabolic index in experimental hyperoxic hypercapnia, hypoxia and ischemia. Stroke 40: 2165-2172, 2009.

16. Malisza K, Kozlowski P, Peeling J, et al: A review of in vivo $1 \mathrm{H}$ magnetic resonance spectroscopy of cerebral ischemia in rats. Biochem Cell Biol 76: 487-496, 1988.

17. Zauner A, Daugherty WP, Bullock MR, et al: Brain oxygenation and energy metabolism: part I-biological function and pathophysiology. Neurosurg 51: 289-301, 2002.

18. Siesjö BK: Pathophysiology and treatment of focal cerebral ischemia. Part II: mechanisms of damage and treatment. J Neurosurg 77: 337-354, 1992.

19. Sun PZ,Zhou J, Huang J, et al: Simplified quantitative description of amide proton transfer (APT) imaging during acute ischemia. Magn Reson Med 57: 405-410, 2007.

20. Sun PZ and Sorensen AG: Imaging pH using the chemical exchange saturation transfer (CEST) MRI: correction of concomitant rf irradiation effects to quantify CEST MRI for chemical exchange rate and pH. Magn Reson Med 60: 390-397, 2008.

21. Jokivarsi KT, Gröhn HI, Gröhn OH, et al: Proton transfer ratio, lactate and intracellular $\mathrm{pH}$ in acute cerebral ischemia. Magn Reson Med 57: 647-653, 2007.

22. Ward KM and Balaban RS: Determination of $\mathrm{pH}$ using water protons and chemical exchange dependent saturation transfer (CEST). Magn Reson Med 44: 799-802, 2000.

23. Zhou J, Payen JF, Wilson DA, Traystman RJ and van Zijl PC: Using the amide proton signals of intracellular proteins and peptides to detect $\mathrm{pH}$ effects in MRI. Nat Med 9: 1085-1090, 2003.

24. Sun PZ, Lu J, Wu Y, Xiao G and Wu R: Evaluation of the dependence of CEST-EPI measurement on repetition time, RF irradiation duty cycle and imaging flip angle for enhanced $\mathrm{pH}$ Sensitivity. Phys Med Biol 58: N229-N240, 2013.

25. Jokivarsi KT, Gröhn HI, Gröhn OH and Kauppinen RA: Proton transfer ratio, lactate and intracellular $\mathrm{pH}$ in acute cerebral ischemia. Magn Reson Med 57: 647-653, 2007.

26. Sun PZ, Cheung JS, Wang E and Lo EH: Association between $\mathrm{pH}$-weighted endogenous amide proton chemical exchange saturation transfer MRI andtissue lactic acidosis during acute ischemic stroke. J Cereb Blood Flow Metab 31: 1743-1750, 2011.
27. Sun PZ, Wang Y, Dai Z, Xiao G and Wu R: Quantitative chemical exchange saturation transfer (qCEST) MRI-RF spillover effect-corrected omega plot for simultaneous determination of labile proton fraction ratio and exchange rate. Contrast Media Mol Imaging 9: 268-275, 2014.

28. Mougin OE, Coxon RC, Pitiot A and Gowland PA: Magnetization transfer phenomenon in the human brain at 7T. NeuroImage 49: 272-281, 2010.

29. Jones CK, Schlosser MJ, van Zijl PC, Pomper MG, Golay X and Zhou J: Amide proton transfer imaging of human brain tumors at 3T. Magn Reson Med 56: 585-592, 2006.

30. Sun PZ, Benner T, Kumar A and Sorensen AG: An investigation of optimizing and translating $\mathrm{pH}$-sensitive pulsed-chemical exchange saturation transfer (CEST) imaging to a 3T clinical scanner. Magn Reson Med 60: 834-841, 2008.

31. Sun PZ, Wang Y, Xiao G and Wu R: Simultaneous experimental determination of labileproton fraction ratio and exchange rate with irradiation radio frequency power-dependentquantitative CEST MRI analysis. Contrast Media Mol Imaging 8: 246-251, 2013.

32. Wu R, Liu CM, Liu PK and Sun PZ: Improved measurement of labile proton concentration-weighted chemical exchange rate (kws) with experimental factor-compensated and T1-normalized quantitative chemical exchange saturation transfer (CEST) MRI. Contrast Media Mol Imaging 7: 384-389, 2012.

33. van Dorsten FA, Olah L, Schwindt W, et al: Dynamic changes of ADC, perfusion and NMR relaxation parameters in transient focal ischemia of rat brain. Magn Reson Med 47: 97-104, 2002.

34. Chen JH, Sambol EB, Decarolis P, O'Connor R, Geha RC, Wu YV and Singer S: High-resolution MAS NMR spectroscopy detection of the spin magnetization exchange by cross-relaxation and chemical exchange in intact cell lines and human tissue specimens. Magn Reson Med 55: 1246-1256, 2006.

35. Makela HI, Kettunen MI, Gröhn OH and Kauppinen RA: Quantitative $\mathrm{T}_{1 \mathrm{r}}$ and magnetic transfer magnetic resonance imaging of acute cerebral ischemia in the rat. J Cereb Blood Flow Metab 22: 547-558, 2002.

36. Dai Z, Ji J, Xiao G, et al: Magnetization transfer prepared gradient echo MRI for CEST imaging. PLoS One 9: e112219, 2014.

37. Bar-Shir A1, Liu G, Greenberg MM, et al: Synthesis of a probe for monitoring HSV1-tk reporter gene expression using chemical exchange saturation transfer MRI. Nat Protoc 8: 2380-2391, 2013.

38. Katsura K, Ekholm A, Anders B and Siesjö BK: Extracellular pH in the brain during ischemia: relationship to the severity of lactic acidosis. J Cereb Blood Flow Metab 11: 597-599, 1991

39. Ward KM and Balaban RS: Determination of $\mathrm{pH}$ using water protons and chemical exchange dependent saturation transfer (CEST). Magn Reson Med 44: 799-802, 2000.

40. Goffeney N, Bulte JW, Duyn J, Bryant LH Jr and van Zijl PC: Sensitive NMR detection of cationic-polymer-based gene delivery systems using saturation transfer via proton exchange. J Am Chem Soc 123: 8628-8629, 2001.

41. Aime S, Delli CD and Terreno E: Highly sensitive MRI chemical exchange saturation transfer agents using liposomes. Angew Chem Int Ed Engl 44: 5513-5515, 2005.

42. Sherry AD and Woods M: Chemical exchange saturation transfer contrast agents for magnetic resonance imaging. Annu Rev Biomed Eng 10: 391-411, 2008.

43. van Zijl PC and Yadav NN: Chemical exchange saturation transfer (CEST): what is in a name and what isn't? Magn Reson Med 65: 927-948, 2011.

44. van Zijl PC, Yadav NN: Chemical exchange saturation transfer (CEST): what is in a name and what isn't? Magn Reson Med 65: 927-948, 2011. 